\title{
OPTICAL PROTEINCHIP AS MICROARRAYS FOR PROTEIN INTERACTION DETERMINATION
}

\author{
G. Jin, Z. H. Wang, Y.H. Meng, P.Q. Ying, and L.H. Xia \\ National Microgravity Laboratory, Institute of Mechanics, Chinese Academy of Sciences, Beijing, P.R.China
}

\begin{abstract}
One kind of optical protein chip and a ProteinChip analysis system have been introduced. The chip is processed with surface patterning, surface modification, ligand immobilization to form a sensing surface with multi-bioactivity. The affinity between proteins is used to realize non-labeling micro-assays for protein identifications and protein interactions. A model of the chip and some demonstration results are presented.
\end{abstract}

Keywords-Protein chip, imaging ellipsometry, protein interaction

\section{INTRODUCTION}

The concept of biosensor based on imaging ellipsometry was presented several years ago $[1,2]$. The further development in protein interaction detection with optical ProteinChip is demonstrated in this report. As an approach to the ProteinChip, the basic idea of the biosensor is repeated at first.

The basic principle is that one kind of optical imaging technique - imaging ellipsometry is used to visualize optical biochips with the lateral thickness (or surface concentration) distribution of protein layers attached on a patterned surface. A ligand and its receptor such as an antibody and its corresponding antigen can assemble into complexes due to their affinity. The optical biosensor is based on that each reactant, referred to as a ligand, is immobilized to a surface as a sensing surface with bioactivity of the ligand. The other reactant, referred to as the analyte (or receptor), exists in solution. The sensing surface is exposed to the solution containing analyte. When the analyte in the solution interacts with its corresponding ligand on the sensing surface and assembles into complex upon their affinity. The layer on the surface where the interaction takes place becomes thicker (or surface concentration higher) than before exposure to the analyte solution. A significant increase of the attached layer thickness (or surface concentration) indicates that the solution contained receptor against the ligand on the surface. With a visualization of imaging ellipsometry, the increase is determined, and in this way, the existence of the analyte in the solution can be verified.

For the optical proteinchip, the substrate of the chip is treated by photolithography or "soft" lithography technique in order to create patterned surface $[3,4]$. Each area of the pattern is corresponding to one bioactivity with ligand immobilization, such as protein immobilization, and the interval between the areas is non-bioactive and optically extinctive. In this case, each area of the proteinchip may function as a biosensor for corresponding analyte (or receptor).

The advantages of the proteinchip is: 1) a multi-detection performed at the same time; 2) the detection without labeling requirements; 3) in-situ and ex-situ detection available for protein analysis; 4) less consume of analytes; 5) quantitative detection with calibrations. Especially it is ideal way to monitor the protein adsorption process and the binding of proteins. Its function of real-time analysis for protein interaction may obtain some important kinetic data such as the protein interaction rate and the interaction conditions, etc. It is not only for one couple of proteins, but also for multicouples available at the same time.

\section{METHODOLOGY}

The proteinchip technology includes several sections: 1) the chip design, which depends on the analytes since each protein has its individual properties; 2) the ligand immobilization, the chip substrate is patterned with photolithography, chemical modification, and adsorption or self-assembly of ligand monolayer, respectively; 3) the cell, in which protein specific binding takes place; 4) the sampling, processing and analyzing the results of protein interactions, respectively; and 5) the database of proteins for further analysis, etc.

The sampling system with imaging ellipsometry has been mentioned in details before [5]. It has the ability to visualize the sub-monolayer of proteins, and the thickness resolution reaches the order of $0.1 \mathrm{~nm}$.

The evolution of the chip is demonstrated in Fig.1. (A) A solid substrate of the biochip is prepared with the technique of photolithograph, similar to which used for microelectronics elements, to create patterned areas and each area isolated with shading background, that is non-bioactive and optically extinctive. Each area surface of the pattern is atomgraded flat like a mirror, and its surface is chemically modified for ligand immobilization. (B) The ligand is immobilized with adsorption or self-assembly to form a sensing surface and the binding sites of ligand exposing to the upside. Various kinds of ligands are pre-attached on each area individually to form sensing areas one by one on the proteinchip surface. (C) After the interaction between proteins, the molecule complexes appear on the chip surface corresponding to analytes in the solution tested. (D) The sampling is performed with the imaging system of in-situ or ex-situ availability. The results are finally grabbed and stored in a computer for further analysis.

For the in-situ sampling, the interaction process between the analytes in solution and the ligands on the surface of patterned biochip can be visualized in one kind of reaction cell specially designed. The cell has a small volume containing the solution and two optical windows for the probe light beam of imaging in and out. The normal of the two 
windows is parallel to the incident direction and the reflection direction of the probe light. The windows with optical quality are transparent for the incident light. The solution could be poured into the cell and pumped out by a micro-fluidics system.

The chip is inserted into the cell. When the solution containing the analytes is poured into the cell, the analytes in the solution will react with their corresponding ligands on the area surface of the chip according to the affinity of protein interaction, so that the thickness (or the surface concentration) of sensing layer in patterned areas increase. The variation process of the layer thickness (or the surface concentration) can be grabbed in a series of images with time, furthermore it is possible to obtain kinetic information for the interactions from a digital image processing.

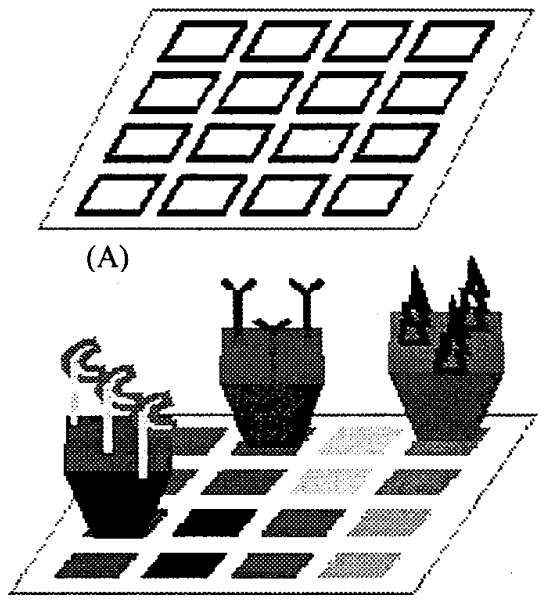

(B)

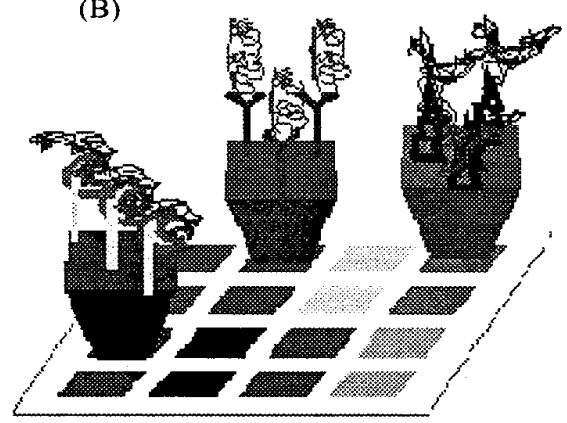

(C)

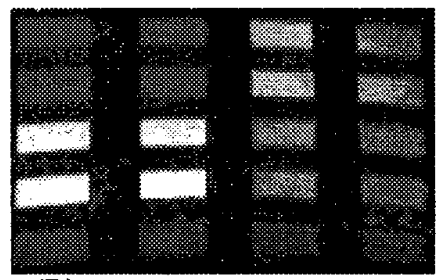

(D)

Fig.1. Proteinchip model

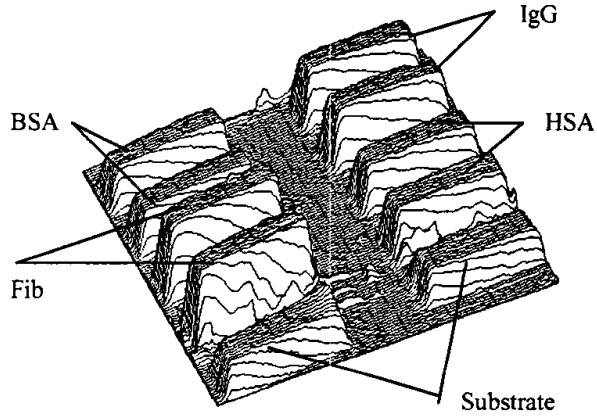

(A)

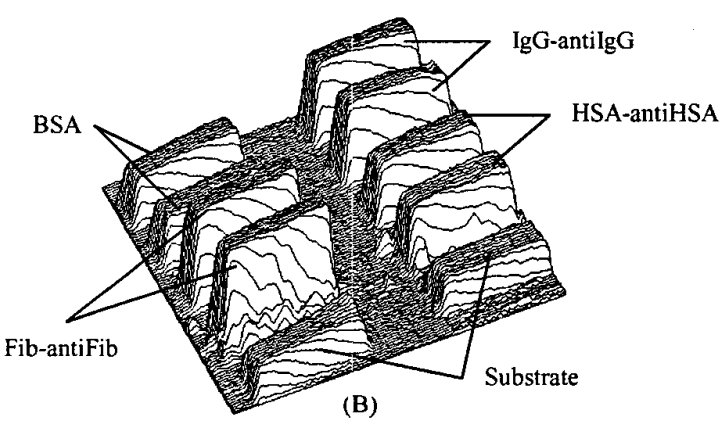

Fig. 2. (A) the chip with BSA, Fib, IgG and HSA layers

(B) the chip after incubation in the serum with antiFib, antilgG and antiHSA.

\section{RESULTS AND DISCUSSION}

A. A proteinchip for multi-protein interactions

A silicon wafer surface was treated with photolithography into ten square areas $\left(1.5 \times 1.5 \mathrm{~mm}^{2}\right)$ separated by the width of $1 \mathrm{~mm}$ of shading background. It was modified with dichlordimethylsilane to prepare for ligand immobilization. There were four kinds of proteins in duplicate immobilized in the areas individually, which were bovine serum albumin (BSA), human fibrinogen (Fib), human immunoglobulin G (IgG), and human serum albumin (HSA), which occupied eight areas and other two bares left as references.

The images sampled are shown in light intensity distribution, which correspond to the sensing layer thickness distribution or the surface concentration of proteins. It is capable of proving

$$
\operatorname{Th}(x, y, t) \propto \sqrt{I(x, y, t)}
$$

that the thickness $T h(x, y . t)$ of the protein layer is proportional to the square root of the intensity $I(x, y, t)[6]$. In this way, the thickness distribution can be shown in three dimensions. The image in fig. 2. (A) shows the thickness distribution of the protein pattern on the chip. The BSA, Fib, IgG, and HSA layers in duplicate as well as the references can be seen. The 
thickness of the HSA layer is almost the same as that of the BSA layer, owing to their similar molecular sizes and same immobilization conditions, the IgG's thicker and the Fib's the thickest. The entire sensing areas of the proteinchip were incubated in serum containing a mixture of antiserum, which contained antibodies of antilgG, antiHSA and antiFib, etc. They reacted with their surface-bound antigens to form complexes IgG-antilgG, HAS-antiHSA and FibantiFib, respectively, so that significant increases of layer thickness in the three couple of areas appeared. These can be obviously seen in Fig. 2 (B), which shows an image taken after the incubation in the antiserum. No significant thickness increase occurred in the BSA areas. Otherwise the sensing surface was incubated in the single antiserum containing antilgG, antiHSA, antiBSA or antiFib, respectively, the corresponding images after incubations in antiserum confirmed the selectivity of such kind of proteinchip.

\section{B. Real-time visualization for multi-process of protein interactions}

On above, the final results for three couples of protein binding according to their affinities were observed on the proteinchip. Furthermore, the dynamic processes of protein interactions became into our interests. For the purpose of real time observation of multi-protein interaction processes, a similar chip with four kinds of ligand immobilized was prepared on the patterned surface. The chip was inserted into the cell containing solution for in-situ experiments and the chip surface with sensing areas was clearly observed. Then the mixture of antiserum containing antibodies of antilgG, antiHSA and antiFib, etc. was poured into the cell. Several binding processes between antigens on the surface and antibodies in the solution were observed simultaneously, which corresponded to the affinities of IgG-antilgG, HSAantiHSA, and Fib-antiFib, etc. respectively. The binding processes resulted in the layer thickness on the corresponding sensing areas increasing with time. Fig.3. just shows a series of thickness variation with time for protein binding processes. These were deduced from the average thickness of corresponding sensing areas during the in-situ experiments. We followed the relationship between the intensity $l(x, y, t)$ and the thickness $\operatorname{Th}(x, y, t)$ of protein layer, and the thickness is proportional to the protein surface density. So the thickness variation means the protein binding to the surface on their affinity. Further observation showed that the binding rate for different protein couple was different from others, even though in the same experimental condition.

The areas where the ligand and receptor reacted into their complexes were chosen for the quantitative result of the interaction process in real-time visualization. The statistical average thickness (in arbitrary unit) over the IgG, HSA and Fib areas are given in Fig. 3. The vertical is the thickness of the sensing layers assembling into complex layer in arbitrary unit, and the horizontal is the time in logarithmic expression. There are two interaction rates for the three binding processes, below and over $100 \mathrm{~s}$ being seen obviously.

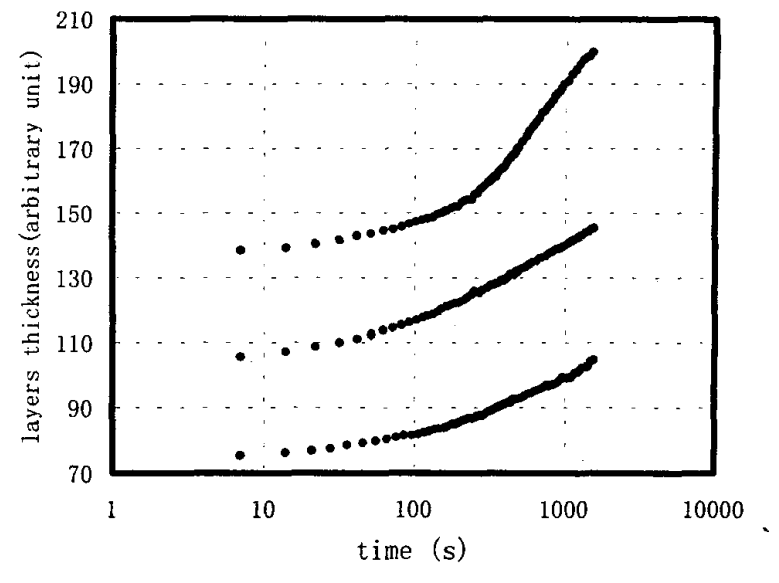

Fig. 3. Fib-antiFib, IgG-antiIgG and HSA-antiHSA binding processes

When the solution with antisera was poured into the reaction cell containing the solution without antisera before, the antibody molecules diffused to everywhere of the cell to reach a uniform distribution of molecule concentration owing to a concentration diffusion. Some antibody molecules diffusing to the sensing surface reacted with the corresponding ligands on the surface binding into their complexes. This was corresponding to the process below 100 s. The diffusion rate that controlled the binding rate is the capital phenomenon. Then the concentration distribution of antibody in the cell reached uniformity. The interaction with a higher rate occurred on whole the sensing surface over $100 \mathrm{~s}$. It showed that the interaction rate is different for the three binding processes. The binding rate between Fib and antiFib is faster than the others, and the slowest one is between HSA and its antibody. Here, only a sketchy description for the protein interaction processes detected at the same time is presented as a demonstration. The further theoretical analysis will be presented in details in other papers.

\section{CONCLUSION}

The above demonstration of the proteinchip for protein interactions shows that the proteinchip is a potential way for multi-protein analysis. It has advantages in simple operation, no labeling, and multi-channel analysis at the same time. It's suitable for micro-assays related to various specific bindings between bio-molecules.

\section{ACKNOWLEDGMENT}

The Chinese Academy of Sciences is acknowledged for its supports.

\section{REFERENCES}


[1] G. JIN, R. Jansson, I. Lundstrom and H. Arwin, "Imaging ellipsometry for biosensor applications", Transducers'95 Eurosensors IX, Stockholm, Sweden, June, 1995.

[2] G. JIN, P. Tengvall, 1. Lundstrom and H. Arwin, "A Biosensor Concep Based on Imaging Ellipsometry for Visualization of Biomolecular Interactions", Analytical Biochemistry, Vol.232, pp.69-72, 1995.

[3] R. S. Kane, et al., "Patteming proteins and cells using soft lithography", Biomaterials, Vol. 20(23-24), pp. 2363-2376, 1999.
[4] H. Fan, et al., "Rapid prototyping of patterned functional nanostructures," Nature, Vol.405 (6782) pp.56-60, 2000

[5] G. JIN, R. Jansson and H. Arwin, "Imaging Ellipsometry Revisited: Developments for Visualization of Thin Transparent Layers on Silicon Substrates," Rev. Sci. Instrum., Vol. 67, pp.2930-2936, 1996.

[6] H. Arwin, S. Welin-Klintström, R. Jansson, "Off-null ellipsometry revisited: basic considerations for measuring surface concentrations a solid/liquid interfaces," J. Colloid Interface Sci., vol. 156, pp. 377-382, 1993. 